\title{
Transtorno de déficit de atenção e hiperatividade: breve revisão teórica no contexto da neuropsicologia infantil
}

Trastorno por déficit de atención e hiperactividad: breve revisión teórica en el contexto de la neuropsicología infantil Le trouble du déficit de l'attention avec hyperactivité : une brève révision de la littérature chez la neuropsychologie de l'enfant Attention-Deficit Hyperactivity Disorder: a brief review in child neuropsychology

\author{
Hosana A. Gonçalves ${ }^{1}$, Janice R. Pureza ${ }^{1}$, \& Mirella L. Prando ${ }^{1}$ \\ ${ }^{1}$ Pontifícia Universidade Católica do Rio Grande do Sul, Brasil.
}

\begin{abstract}
Resumo
O Transtorno de Déficit de Atenção e Hiperatividade (TDAH) é, entre os distúrbios do desenvolvimento, um dos diagnósticos mais realizados na atualidade, com uma alta prevalência na população infantil e uma das principais causas de procura por atendimentos em ambulatórios de saúde mental por parte de crianças e adolescentes. Este artigo tem por objetivo apresentar uma breve revisão teórica da literatura sobre o TDAH no contexto clínico e neuropsicológico. Fez-se uma revisão não sistemática em periódicos e bases de dados brasileiras e internacionais. Observam-se déficits cognitivos em crianças com TDAH, entre dificuldades de atenção, dememória de trabalho e de funções executivas (FE), ocasionando prejuízo em situações de tomada de decisões e automonitoramento. Parece haver um crescimento do uso de técnicas e instrumentos específicos de avaliação neuropsicológica para o exame das funções cognitivas envolvidas no TDAH. Essas ferramentas são importantes para a compreensão sobre a neuropsicologia desta patologia, assim como para o diagnóstico e tratamento do TDAH

Palavras-chave: TDAH; infância; neuropsicologia; testes neuropsicológicos.
\end{abstract}

\section{Resumen}

El Trastorno por Déficit de Atención e Hiperactividad (TDAH) es, entre los trastornos del desarrollo, uno de los cuadros más diagnosticados en al actualidad, con una alta prevalencia en población infantil. Constituye una de las principales causas de consulta en centros ambulatorios de salud mental. El objetivo de este artículo es presentar una breve revisión teórica de la literatura sobre TDAH en el contexto clínico y neuropsicológico. Se llevó a cabo una revisión no sistemática en revistas y bases de datos locales y extranjeras. Se ha observado que los niños con TDAH manifiestan déficits cognitivos que afectan la atención, la memoria de trabajo y las funciones ejecutivas y que causan dificultades en situaciones en las que se requiere de toma de decisiones y automonitoreo. Parece existir un uso creciente de técnicas e instrumentos específicos de evaluación neuropsicológica de las funciones cognitivas implicadas en el TDAH. Dichas herramientas son importantes para comprender los aspectos neuropsicológicos de esta patología así como para el diagnostico y tratamiento de esta condición.

Palabras-clave: TDAH; infancia; neuropsicología; test neuropsicológicos.

\section{Résumé}

Le trouble du déficit de l'attention avec hyperactivité (TDAH) est l'une des plus fréquentes diagnoses faites dans nos jours avec une grande prévalence chez l'enfant. Il est aussi l'une des causes majeures de consultation en santé mentale des enfants et des adolescents. Ses caractéristiques principales comprennent des déficits de l'attention avec hyperactivité / comportements impulsifs qui causent des troubles psychologiques et émotionnels. Les troubles cognitifs chez l'enfant atteint de TDAH, la mémoire de travail et les fonctions exécutives (FE) y comprises, provoquent des difficultés dans des situations de prise de décision et du «self-monitoring ». L'utilisation des techniques et des outils spécifiques pour l'évaluation neuropsychologique des fonctions cognitives impliquées dans le TDAH est important pour comprendre les caractéristiques neuropsychologiques de cette condition. Cet article a pour but de présenter une brève révision théorique de la littérature du TDAH dans les contextes clinique et neuropsychologique.

Mots-clés: TDAH; enfance; tests neuropsychologiques.

Artigo recebido: 25/10/2011; Artigo revisado: 17/12/2011; Artigo aceito: 28/12/2011.

Hosana Alves Gonçalves, Faculdade de Psicologia, Pontifícia Universidade Católica do Rio Grande do Sul. Janice da Rosa Pureza, Faculdade de Psicologia, Pontifícia Universidade Católica do Rio Grande do Sul. Mirella Liberatore Prando, Faculdade de Psicologia, Pontifícia Universidade Católica do Rio Grande do Sul.

Correspondências relacionadas a esse artigo devem ser enviadas a: Hosana Alves Gonçalves. Endereço: Av. Ipiranga, 6681, Prédio 11, sala 932, Partenon, Porto Alegre, Brasil, CEP 90619-900.

E-mail: hosana.goncalves@ acad.pucrs.br

DOI: $10.5579 / \mathrm{rnl} .2011 .0076$ 
Attention Deficit Hyperactivity Disorder (ADHD) is one of the most common diagnoses made today with a high prevalence in children and one of the main causes for seeking care in outpatient mental health by children and adolescents. The main characteristics are the deficits in attention and hyperactivity / impulsivity behaviors, causing emotional and psychosocial damage. Cognitive deficits are also observed in children with ADHD, including the working memory and executive functions (EF), causing difficulties in situations of making decision and self-monitoring. The use of specific techniques and instruments for examining neuropsychological assessment of cognitive functions involved in ADHD are important for understanding the neuropsychology of this pathology, as well as for diagnosis and treatment of this condition. This article aims to present a brief theoretical review of the literature on ADHD in clinical and neuropsychological context. Keywords: ADHD; childhood; neuropsychology; neuropsychological tests.

O atualmente denominado Transtorno de Déficit de Atenção/Hiperatividade (TDAH), nem sempre recebeu este nome. Sob uma perspectiva histórica, por volta da metade do século XIX, a primeira referência na literatura médica usada para denominar o quadro foi "transtornos hipercinéticos" (Catelan-Mainardes, 2010; Rohde, et al., 1998). Embora já descrita há muito tempo, apenas na década de 40, foi designada como "lesão cerebral mínima". O uso deste termo se deu pelo fato das evidências da época sugerirem que as alterações comportamentais estavam associadas a lesões no sistema nervoso central provocadas por doenças causadas por vírus, traumatismos cranianos ou acidentes perinatais (Couto, Melo-Junior, \& Gomes, 2010; Possa, Spanemberg, \& Guardiola, 2005; Rohde, et al., 1998; Taylor, 2010).

Em 1962, passou-se a utilizar o termo "disfunção cerebral mínima", reconhecendo-se que as alterações características da síndrome relacionavam-se mais a disfunções nas vias nervosas do que propriamente a lesões nas mesmas. Ainda na década de 1960, a segunda edição do DSM (Manual de Diagnóstico e Estatístico das Perturbações Mentais) utilizou o termo "Reação Hipercinética" para descrever a síndrome. Na década de 70, a CID-9 (Código Internacional de Doenças) usou o termo "Síndrome Hipercinética". Uma década mais tarde, quando foi publicado o DSM-III, utilizavase o termo "Distúrbio de Déficit de Atenção" (DDA), já nesta versão o déficit podia ser acompanhado ou não de hiperatividade. Os sistemas classificatórios atuais utilizados em psiquiatria usam o termo "Transtornos Hipercinéticos" (CID-10) e o DSM-IV-TR (APA, 2002) denomina "Transtorno por Déficit de Atenção/Hiperatividade" (CatelanMainardes, 2010).

A prevalência do TDAH no Brasil é alta, sendo que em crianças em idade escolar, a prevalência média varia de 5 a $18 \%$, podendo a mesma variar conforme a região do país e a forma como a avaliação diagnóstica é realizada, além da faixa etária avaliada (Guardiola, Fuchs, \& Rotta, 2000; Possa, Spanemberg, \& Guardiola, 2005; Costa, Maia Filho, \& Gomes, 2009; Charach, 2010; Fontana, et al., 2007). Outro dado é que uma das principais causas de procura de atendimento em ambulatórios de saúde mental, por parte de crianças e adolescentes, ocorre por dificuldades que representam a sintomatologia do TDAH (Faraone, et al., 2003).

Juntamente com as dificuldades em decorrência da falta de concentração e da hiperatividade/impulsividade, o indivíduo portador do transtorno pode vivenciar ainda uma série de outros prejuízos como problemas emocionais e abuso de substâncias na adolescência (Charach, 2010; Glass, et al., 2011). Com o avanço da idade, observa-se também o aumento de comorbidades, como transtornos neurológicos e neuropsiquiátricos, bem como transtornos de aprendizagem (dislexia e discalculia, principalmente), transtornos do humor e de ansiedade, transtornos disruptivos, comportamento suicida, transtorno de personalidade anti-social na adultez, tiques, dificuldades no sono, enurese, além de epilepsias (Catelan-Mainardes, 2010; Charach, 2010; Glass, et al., 2011; Costa, Maia Filho, \& Gomes, 2009; Mayes, et al., 2009; Rohde, et al., 2004).

Alguns estudos demonstram que o TDAH, em suas subdivisões propostas pelo DSM-IV-TR (APA, 2002) - tipos com predomínio de desatenção, combinado e/ou hiperatividade/impulsividade - apresenta algumas diferenças relacionadas ao impacto de suas características, sendo o tipo desatento mais associado ao comprometimento escolar e neuropsicológico, e o tipo hiperativo/impulsivo mais relacionado a dificuldades comportamentais (Coutinho, Mattos e Araújo, 2007). Este quadro desencadeia problemas não somente à criança, mas para as suas redes sociais, incluindo familiares e o meio escolar, sendo esse impacto, inclusive, um dos fatores diagnósticos para o TDAH. Além disso, o transtorno afeta de modo adverso o ajustamento psicossocial da criança, o que cria a necessidade de algum tipo de intervenção (Possa, Spanemberg, \& Guardiola, 2005).

Devido ao grande impacto originado por este quadro clínico, o presente artigo busca apresentar um breve apanhado a respeito da neuropsicologia do TDAH e dos principais instrumentos de avaliação neuropsicológica utilizados na sua avaliação. Para isso fez-se uma revisão não sistemática em periódicos e bases de dados brasileiras e internacionais.

\section{Características gerais do TDAH}

Dentre as características principais que devem estar presentes necessariamente para o diagnóstico de TDAH, segundo o DSM-IV-TR (APA, 2002) citam-se: a) iniciar os sintomas antes dos 7 anos de idade; b) persistir por pelo menos 6 meses consecutivos; c) estar presente em dois ou mais ambientes e; 4) causar prejuízo funcional nos contextos social, acadêmico ou ocupacional (APA, 2002). A oscilação do comportamento é uma marcante característica dessas crianças (Couto, Melo-Junior, \& Gomes, 2010), sendo que a principal refere-se a um padrão persistente de falta de atenção e/ou hiperatividade, mais freqüente e intenso que aquele apresentado por indivíduos na mesma faixa de desenvolvimento (Fontana, et al., 2007).

O transtorno pode apresentar-se em três subtipos: (1) Tipo Predominantemente Desatento; (2) Tipo Predominantemente Hiperativo-Impulsivo e (3) Tipo Combinado, sendo que neste último tanto sintomas de desatenção como sintomas de hiperatividade/impulsividade estavam presentes, de maneira significativa, nos últimos seis meses (APA, 2002). Ainda no subtipo combinado, a hiperatividade se manifesta não só como inquietação motora, mas também como inquietação intelectual e verbal. A impulsividade é evidenciada por respostas aceleradas, 
dificuldade de autocontrole e de auto-regulação, de seguir ordens seqüenciais e de antecipar as consequências de seus atos. Juntamente com isso, a pessoa se distrai com facilidade diante do menor estímulo, interrompendo continuamente suas atividades (Couto, Melo-Junior, \& Gomes, 2010).

O tipo com predomínio de sintomas de desatenção é marcado pela dificuldade para ater-se aos detalhes, ocasionando assim erros grosseiros nas atividades que executa e falta de organização, dificultando a conclusão das atividades. Parecem, ainda, não escutar o que lhes é falado diretamente (Rohde, et al., 2004).

No tipo predominantemente hiperativo/impulsivo predomina a agitação psicomotora, a fala em demasia, o indivíduo tem baixa tolerância à frustração, tem dificuldade de esperar sua vez para falar e é extremamente solicitante. A rejeição dos pares e a maior suscetibilidade a acidentes com ferimentos são frequientes neste subtipo do transtorno, além de que crianças com este subtipo apresentam dificuldades de esperar em situações onde cada um tem sua vez (APA, 2002). No que se refere aos estudos de genética do TDAH, alguns destes sugerem alto grau de hereditariedade dos sintomas (Charach, 2010). Ressalta-se ainda que alguns eventos pré ou perinatais podem interferir no amadurecimento neurológico normal, como, por exemplo, o baixo peso ao nascer e a exposição ao álcool ou cigarros pela mãe durante a gestação, aumentando, assim, o risco para o desenvolvimento do TDAH (Catelan-Mainardes, 2010). Assim, a etiologia desse transtorno é considerada neuro-genético-ambiental (Fontana, et al, 2007; Couto, Melo-Junior \& Gomes, 2010) .

Os circuitos neuronais associados com o transtorno incluem o córtex pré-frontal, gânglios da base e cerebelo (Szobot et al., 2001; Catelan-Mainardes, 2010). Em relação aos sistemas de neurotransmissores envolvidos, há indicações na literatura sobre o envolvimento das catecolaminas, sugerindo disfunções na transmissão de dopamina e/ou noradrenalina (Couto, Melo-Junior, \& Gomes, 2010; Lufti \& Carvalho, 2010; Rohde, et al., 1998). Especificamente, as insuficiências nos circuitos do córtex pré-frontal e amígdala, a partir da neurotransmissão das catecolaminas, resultam nos sintomas de esquecimento, distratibilidade, impulsividade e desorganização (Couto, Melo-Junior \& Gomes, 2010).

Segundo a Associação Brasileira do Déficit de Atenção (ABDA), o tratamento para o TDAH deve ser multidisciplinar, devendo envolver não só o indivíduo, mas também sua família, sua escola e profissionais de saúde da comunidade. $O$ tratamento deve combinar a intervenção farmacológica com orientações para a família e escola acerca de como lidar com a pessoa com TDAH. É extremamente importante que o professor saiba utilizar técnicas específicas para o manejo de alunos com esse transtorno em sala de aula. Além disso, existem estratégias que, quando ensinadas ao portador, mostram-se muito eficazes e podem ser ensinadas em psicoterapia, minimizando suas dificuldades. Um dos tratamentos psicoterápicos indicados para o tratamento do TDAH é a Terapia Cognitivo-Comportamental, uma vez que esta modalidade terapêutica apresenta evidências científicas para lidar com sua sintomatologia (ABDA, 2010; Rohde \& Halpern, 2004).

É importante ressaltar que o TDAH não é em si um transtorno de aprendizagem, mas os sintomas deste transtorno podem vir acompanhados por dificuldades nessa área, como, por exemplo, quadros de dislexia e disortografia, sendo recomendado o auxílio de um fonoaudiólogo (ABDA, 2010).
Em relação à medicação, as intervenções psicofarmacológicas devem ser cuidadosamente avaliadas por um médico especialista, uma vez que mais de $70 \%$ das pessoas com TDAH que procuram atendimento ambulatorial especializado na área podem apresentar algum tipo de comorbidade (Souza, et al., 2004) como transtornos de humor e de ansiedade (Sarkis, Sarkis, Marshall, \& Archer, 2005), transtornos de conduta (Charach, 2010), entre outros quadros (Glass, Flory, Martin, \& Hankin, 2011; Sobanski, Banaschewski, Asherson, Buitelaar, Chen, et al., 2010).

\section{Neuropsicologia do TDAH Infantil}

O funcionamento neuropsicológico reflete o desenvolvimento cognitivo, psicossocial e comportamental dos indivíduos. A compreensão dessa relação é de extrema importância para o diagnóstico e tratamento de diversos transtornos comportamentais que podem ocorrer na infância (Ellison \& Semrud-Clickiman, 2007; Lezak, Howieson, \& Loring, 2004). Para isso, a avaliação neuropsicológica utilizase de técnicas e instrumentos validados para mensurar os processamentos envolvidos, além da observação e entrevista clínicas. Tem como objetivo identificar e descrever os padrões de funcionamento cognitivo esperado de acordo com o nível sociocultural, desenvolvimental e outras diferenças individuais da população. Para isso, tornam-se necessários instrumentos específicos e métodos de investigação sensíveis para o exame dessas funções cognitivas (Andrade, 2002; Argolo, Bueno, Shaver, Godinho, Abreu, Duran, et al., 2009).

Estudos sugerem que a maioria das crianças com TDAH demonstram alguns prejuízos cognitivos como em funções executivas, memória de trabalho e atenção (Charach, 2010; Rohde, 1998). Elas podem apresentar dificuldades em situações novas que exigem a tomada de decisão e de automonitoramento, compatível com as condições sob as quais o componente executivo central, teoricamente, é ativado (Oliveira, 2007). Estes achados sugerem que os prejuízos subjacentes a estas alterações podem estar relacionados aos danos observados na vida diária dos portadores do transtorno.

Um estudo de Rizzutti e colaboradores (2008), que objetivou delimitar indicadores clínicos e neuropsicológicos em 150 crianças com suspeita de TDAH, foram encontrados prejuízos nas habilidades visuoconstrutivas, na memória visuo-espacial, na capacidade de inibição motora, de planejamento e de organização acessadas pelas Figuras Complexas de Rey, tanto no grupo de pacientes com tipo combinado do déficit quanto no predominantemente desatento quando comparados ao grupo controle. Já o desempenho no Conners' Continuous Performance Test (CPT) evidenciou que o grupo clínico apresentou um tempo de reação mais lento, mais erros de omissão e comissão, maior variabilidade de resposta e alto grau de perseveração quando comparados ao grupo controle. Também foram encontrados prejuízos na memória de trabalho através dos escores no subteste Dígitos do WISC-III e dos Blocos de Corsi.

Estudos internacionais sugerem que 25 a $50 \%$ das crianças com TDAH demonstram déficit inibitório e este percentual não é diferente nos subtipos do transtorno, sendo uma das causas de dificuldades atencionais e de processamento executivo (Barkley, 1997; Wahlstedt, 2009). Além de falhas importantes nas funções cognitivas citadas, uma revisão feita por Silva e Souza (2005) refere a possibilidade de atraso na aquisição da linguagem, trocas fonêmicas e dificuldade de coordenação motora em crianças 
portadoras, pelo fato de a atenção desempenhar papel fundamental no processamento da linguagem oral e escrita.

Levando-se em consideração as diversas alterações neuropsicológicas que podem ocorrer em pacientes com TDAH, é fundamental que as práticas clínicas contemplem cada vez mais a investigação específica a fim de promover o entendimento de como as dificuldades em funções cognitivas se relacionam e se manifestam nesse transtorno. Este entendimento possibilitará um planejamento terapêutico específico e eficaz para cada caso.

Apesar do TDAH ser caracterizado pelos sintomas de desatenção, hiperatividade e impulsividade, trata-se de uma patologia heterogênea, que provoca amplo prejuízo no funcionamento cognitivo e interpessoal. Esta condição clínica inclui, principalmente, déficits atencionais e de funções executivas devido a alterações no sistema dopaminérgico, causando uma hipofunção dos circuitos frontais corticais e sub corticais (Clark, Prior \& Kinsella, 2000).

\section{Avaliação neuropsicológica do TDAH Infantil}

Pela presença de alterações cognitivas neste quadro, salienta-se a importância de estudos investigativos de avaliação neuropsicológica. Neste contexto, a seleção de testes adequados para avaliar as funções cognitivas envolvidas de forma específica, torna-se relevante para a determinação de um perfil de dados precisos sobre o funcionamento e o comportamento do indivíduo.

Embora o TDAH seja um transtorno popularmente conhecido pelo déficit de atenção, ressalta-se que a atenção é uma função multifacetada e intrinsecamente relacionada a outras funções tais como os processos executivos e à inteligência (Costa, Maia Filho \&Gomes, 2009). O estudo dos subcomponentes das funções executivas vem tomando grande proporção e relevância nas pesquisas em TDAH, uma vez que demonstram apresentar forte impacto e relação com este quadro clínico (Shuai, Chan \& Wang, 2011). Além disso, transtornos específicos de linguagem e de aprendizagem são comuns neste grupo clínico (Charach, 2010; Jonsdottir, Bouma, Sergeant, \& Scherder, 2006; Mayes et al, 2009; Silva e Souza, 2005; Wu, Anderson, \& Castiello, 2002).

Nesse contexto, os instrumentos de avaliação neuropsicológica do TDAH podem ser utilizados isoladamente, com interesses específicos ou fazer parte de baterias completas. Assim, vem sendo utilizados testes que mensuram atenção concentrada (Continuous Performance Test, Teste de Atenção Visual-III, Teste D2 e o Teste de Atenção concentrada - AC), testes de mensuração de subcomponentes das funções executivas (Torre de Hanói, testes de fluência verbal, Teste Stroop de Cores e Palavras, Teste Wisconsin de classificação de Cartas entre outros) (para uma revisão mais específica concusultar Fonseca et al, 2010).

Porém, assim como a atenção, o termo funções executivas se trata de um construto teórico multidimensional cujos componentes ainda não foram identificados nem delimitados claramente (Soprano, 2003) e existe uma escassez de estudos publicados que tenham avaliado cada subcomponente específico das FE tanto em nível nacional quanto internacional, situação essa que pode dificultar uma maior elucidação a cerca da compreensão de todos os aspectos envolvidos nesse quadro clínico.

Nesse sentido, alguns estudos utilizam instrumentos que avaliam atenção e memória (de trabalho, visuoconstrutiva) (Mayes et al., 2008; Mullane, et al., 2010; Rizzutti, 2008; Rubio et al., 2011) entre crianças com TDAH e controles saudáveis, ou comparando os subtipos desatento e hiperativo do transtorno. Os resultados destes estudos sugerem que este grupo clínico tem prejuízos significativos nas funções cognitivas examinadas. Um estudo de Wahlstedt (2009) avaliou inibição (através dos paradigmas Stroop e Go/noGo), tempo de reação (Go/noGo) e tomada de decisão (Flower-Delay task, Star-Delay card game) em portadores de TDAH com ou sem sinais de transtorno desafiador opositivo (TDO) em comorbidade. Os resultados deste estudo demonstram que independente de o TDO estar presente, os déficits inibitórios são alterações evidenciadas em crianças com TDAH.

Seguindo no contexto da avaliação neuropsicológica do TDAH infantil, Sarkis, Sarkis, Marshal e Archer (2005) utilizou a tarefa Tower of London para avaliar se comorbidades como transtornos de humor, de ansiedade e desafiador opositivo podem interferir na performance de crianças com TDAH nesta tarefa que, segundo os autores, se propõe a avaliar planejamento, memória de trabalho e antecipação. Os resultados apontam para uma não interferência das comorbidades no desempenho executivo do grupo avaliado. Nesta mesma linha, Padolsky (2008) estudou as conseqüências de TDAH em comorbidade com dificuldades de aprendizagem e otite média. A autora utilizou o NEPSY para avaliar processamento fonológico, compreensão e repetição de sentenças e ainda, avaliou a velocidade de processamento auditivo da informação através do IVA. Os resultados sugerem que o TDAH comórbido com otite média pode agravar os déficits de aprendizagem.

O estudo de Shuai, Chan \& Wang (2011) encontrou déficits em funções executivas em crianças com TDAH em comorbidade com os Transtornos de Aprendizagem e, afirma que, principalmente diante deste tipo de combinação de quadros os déficits no componente de inibição são mais severos, devendo ser mais investigados. Percebe-se, assim, tentativas importantes na literatura com objetivo de melhor compreender todas as facetas desse fenômeno que atinge uma considerável parcela da população.

\section{Conclusão}

O TDAH é um dos diagnósticos mais realizados na atualidade, sendo considerável o número de crianças com este transtorno, principalmente, no âmbito escolar. Mesmo sendo caracterizada pelos sintomas de desatenção, hiperatividade e impulsividade, o TDAH é uma patologia heterogênea, que provoca amplo prejuízo no funcionamento cognitivo e interpessoal.

Esta condição clínica inclui, ainda, déficits cognitivos, como dificuldades atencionais, de linguagem, déficits no processamento executivo, ou seja, no conjunto de processos que envolvem memória de trabalho, capacidade de planejamento e organização, flexibilidade cognitiva, controle inibitório, enfim, habilidades fundamentais para o processo de tomada de decisões, controle das emoções e comportamento social. Muitos estudos e teorias procuram explicações para a compreensão deste transtorno que é responsável por dificuldades e sofrimento na vida dos seus portadores e das funções cognitivas envolvidas nesse fenômeno.

Esta revisão teórica não sistemática procurou fazer uma breve descrição da neuropsicologia do TDAH e dos instrumentos utilizados para a avaliação neuropsicológica de 
crianças acometidas pelo transtorno. Observa-se que embora a neuropsicologia do TDAH venha se desenvolvendo cada vez mais em âmbito internacional e nacional, ainda é necessário um entendimento mais profundo sobre a relação entre funções cognitivas em crianças com esse diagnóstico.

\section{Referências}

American Psychiatric Association - APA. (2002). Manual Diagnóstico e Estatístico de Transtornos Mentais. Trad. Cláudia Dornelles. 4 edição revisada. Porto Alegre: Artmed.

Andrade, V. M. (2002). Das Bases Históricas da Neuropsicologia à Avaliação Psicológica. In R.M. Cruz, J. C. Alchieri, J. R. \& J. Sardá Medidas psicológicas: Produção do Conhecimento e da Intervenção Profissional. São Paulo: Casa do Psicólogo, 35-39.

Argolo, N., Bueno, O. F. A., Shaver, B., Godinho, K., Abreu, K., Duran, P., Assis, A., Lima, F., Silva, T., Guimarães, J., Carvalho, R., Moura, I., \& Seabra, A. G. (2009). Adaptação Transcultural da Bateria Nepsy Avaliação Neuropsicológica do Desenvolvimento: Estudo-Piloto. Avaliação Psicológica, 8(1), pp. 59-75

Associação Brasileira do Déficit de Atenção - ABDA. Transtorno do Déficit de Atenção/Hiperatividade: Tratamento. Disponível em: http://www.tdah.org.br/br/sobre-tdah/tratamento.html Acesso em: 24.05.11.

Barkley, RA. (1997). Behavioral inhibition, sustained attention, and executive functions: constructing a unifying theory of ADHD. Psychological Bulletin, 121 (1): 65-94,

Barkley, R. A., Fischer, M., Smallish, L., \& Fletcher, K. (2006). Young adult outcome of hyperactive children: Adaptive functioning in major life activities. Journal of the American Academy of Child and Adolescent Psychiatry, 45 (2), 192 - 202.

Catelan-Mainardes, S. C. (2010). Transtorno de Déficit de Atenção e hiperatividade na infância e adolescência pela perspectiva da neurobiologia. Saúde e Pesquisa, 3 (3), 385-391.

Charach, A. (2010). Children with Attention Deficit Hiperactivity Disorders: Epidemiology, Comorbidity and assessment. Encyclopedia on Early Childhood Development, 1-11.

Costa, C. R. C. M., Maia Filho, H. S., Gomes, M. M. (2009). Avaliação Clínica e Neuropsicológica da Atenção e Comorbidade com TDAH nas Epilepsias da Infância: Uma revisão sistemática. Journal Epilepsy Clinical Neurophysiology, 15 (2), 77-82.

Coutinho, G.; Mattos, P.\&, Araújo, C. (2007). Desempenho neuropsicológico de tipos de transtorno de déficit de atenção e hiperatividade (TDAH) em testes de atenção visual. Jornal Brasileiro de Psiquiatria, 56 (1), 13-16.

Couto, T. S., Melo Junior, M. R., Gomes, C. R. A.(2010). Aspectos neurobiológicos do transtorno do déficit de atenção e hiperatividade (TDAH): uma revisão. Ciências \& Cognição, 15 (1), 241-251.

Ellison, P. A. T., \& Semrud-Clickiman, M. (2007). Child Neuropsychology: Assessement and Interventions for Neurodevelopmental Disorders. New York: Springer Science + Bussiness Media, 300 p.

Fonseca, R. P., Zimmermann, N., Bez, M. B., Willhelm, A., SchneiderBakos, D. (2011). Avaliação neuropsicológica no TDAH e implicações para a terapia cognitivo-comportamental. In: Petersen. C. S., Weiner, R. e Cols. Terapias Cognitivo-Comportamentais para Crianças e Adolescentes - Ciência e Arte. Artmed: Porto Alegre, 400 pg.

Fontana, R.S, Vasconcelos, M.M, Werner Jr., J., Góes, F.V., Liberal, E.F. (2007). Prevalência de TDAH em quatro escolas públicas brasileiras. Arquivos de Neuropsiquiatria, 65 (1), 134-137.

Fontenelle, L. (2001). Neurologia na adolescência. Jornal de Pediatria, 77 (2), 205-216

Guardiola, A., Fuchs, F. D., Rotta, N. T. (2000). Prevalence of AttentionDeficit Hyperactivity disorders in students: comparison between DSM-IV and Neuropsychological criteria. Arquivo Neuropsiquiatria, 58(2-B), 401-407.

Glass, K., Flory, K., Martin, A., Hankin, B. L. (2011). ADHD and comorbid conduct

problems among adolescents: associations with self-esteem and substance use Attention Deficity Hyperactivity Disorder, 3(1), 29-39.

Lezak, M. D., Howieson, D. B., \& Loring, D. W. (2004). Neuropsychological Assessment. New York: Oxford Univertsity Press.

Loutfi, K.S. \& Carvalho, A.S. (2010). Possible interfaces between attention deficit hyperactivity disorder and epilepsy. Jornal Brasileiro de Psiquiatria, 59 (2)

Mayes, S. D., Calhoun, S. L., Chase, G. A., Mink, D. M., Stagg, R. E. (2009). ADHD Subtypes and Co-Occurring Anxiety, Depression, and Oppositional-Defiant Disorder: Differences in Gordon Diagnostic
System and Wechsler Working Memory and Processing Speed Index Scores. Journal of Attention Disorders, 12 (6), 540-550.

Mullane, J. C., Corkum, P. V., Klein, R. M., McLaughlin, E. N., \& Lawrence, M. A. (2010). Alerting, Orienting, and Executive Attention in Children with ADHD. Journal of Attention Disorders, 20 (10), 1-12.

Oliveira, R. M. (2007). O conceito de executivo central e suas origens. Psicologia: Teoria e Pesquisa, 23(4), 399-406.

Padolsky, I. (2008). The Neuropsychological and Neurobehavioral Consequences of ADHD Comorbid with LD and Otitis Media. Journal of Developmental and Physical Disabilities, 20, 11-20.

Possa, M. A., Spanemberg, L., Guardiola, A. (2005). Comorbidades do transtorno de Déficit de atenção e hiperatividade em crianças escolares. Arquivo Neuropsiquiatria, 63(2-B), 479-483.

Rizzuti, S., Sinnes, E. G., Scaramuzza, L. F., Freitas, L., Pinheiro, D., Palma, S. M., Mello, C. B., Miranda, M. C., Bueno, O. F. A., \& Muszkat, M. (2008). Clinical and Neuropsychological profile in a sample of Children with Attention Deficit Hyperactivity Disorders. Arquivos de Neuropsiquiatria, 66(4), 821-827.

Rohde, L. A., Busnello, E. D., Chachamovich, E., Vieira, G. M., Pinzon, V., Ketzer, C. R. (1998). Transtorno de déficit de atenção/hiperatividade: revisando conhecimentos. Revista Brasileira de Psiquiatria, 20 (4), 166-178.

Rohde, L. A., Constantino, E., Miguel Filho, Benetti, L. , Gallois, C., Kieling, C. (2004). Transtorno de déficit de atenção/hiperatividade na infância e na adolescência: considerações clínicas e terapêuticas. Revista Psiquiatria Clínica, 31 (3), 124-131.

Rohde, A. \& Halpern, R. (2004). Transtorno de déficit de atenção/hiperatividade: atualização. Jornal de Pediatria, 80 (2).

Rubio, B., Hernández, S., Verche, E., Martín, R., González-Pérez, P. (2011). A pilot study: differential effects of methylphenidate-OROS on working memory and attention functions in children with attentiondeficit/hyperactivity disorder with and without behavioural comorbidities. ADHD Attention Deficit and Hyperactivity Disorders, 3, 13- 20.

Sarkis, S. M., Sarkis,E. H., Marshall, D., Archer, J. (2005). Self-Regulation and Inhibition in Comorbid ADHD Children: An Evaluation of Executive Functions. Journal of Attention Disorders, 8 (3), 96-108.

Sesma, H. W., Mahone, E. M., Levine, T., Eason, S. H., Cutting, L. E. (2009). The contribution of executive skills to reading comprehension. Child Neuropsychology, 15, 232-246.

Silva, R. A., Souza, L. A. P.(2005). Aspectos Lingǘsticos e Sociais Relacionados ao Transtorno de Déficit de Atenção/Hiperatividade. Revista CEFAC, 7(3), 295-299.

Shuai, L,. Chan, R.C.K., \& Wang, Y. (2011). Executive function profile of chinese boys with attention-deficit hyperactivity disorder: different subtypes and comorbidity. Archives of Clinical Neuropsychology, $26,120-132$.

Sobanski, E., Banaschewski, T., Asherson, P., Buitelaar, J., Chen, W., Franke, B., Holtmann, M., Krumm, B., Sergeant, J., Sonuga-Barke, E., Stringaris, A., Taylor, E., Anney, R., Ebstein, R. P., Gill, M., Miranda, A., Mulas, F., Oades, R. D., Roeyers, H., Rothenberger, A., Steinhausen, H.-C., \& Faraone, S. V. (2010). Emotional lability in children and adolescents with attention deficit/hyperactivity disorder (ADHD): clinical correlates and familial prevalence. Journal of Child Psychology and Psychiatry, 51,915-923.

Soprano, A. M. (2003). Evaluación de las funciones ejecutivas en el niño. Revista Neurologia, 37 (1), 44-50.

Souza, L., Pinheiro, M.A., Denardin, D., Mattos, P., Rohde, L.A. (2004). Attention-deficit/hyperactivity Disorder and Comorbidity in Brazil: Comparison between Two Referred Samples. European Child Adolescents Psychiatry, 13 (4), 243, 248.

Szobot, C., Eizirik, M., Cunha, R. D., Langleben, D., \& Rohde, L. A. (2001). Neuroimagem no transtorno de déficit de atenção/hiperatividade. Revista Brasileira de Psiquiatria, 23(1), 32-35.

Taylor, E. (2010). Antecedents of ADHD: a historical account of diagnostic concepts. ADHD Attention Deficit and Hyperactivity Disorders.

Wahlstedt, C. Neuropsychological Deficits in Relation to Symptoms of ADHD: Independent Contributions and Interactions. Child Neuropsychology, 15 (3), 262- 279.

Wu, K. K., Anderson, V., \& Castiello, U. (2002). Neuropsychological evaluation of deficits in executive functioning for ADHD children with or without learning disabilities. Developmental Neuropsychology, 22 (2), $501-531$. 